\title{
Teste de avaliação in vitro e criopreservação do sêmen de cão utilizando diferentes diluidores
}

\section{In vitro evaluation test and dog semen's freeze with different extenders}

\author{
Cristiane Scavuzzi Moura, ${ }^{*}$ Márcia Cristina Oliveira Cavalcanti, ${ }^{*}$ Maria Madalena Pessoa Guerra, ${ }^{\star}$ André Mariano Batista, ${ }^{\star}$ \\ Márcia Brayner Paes Barreto*
}

\begin{abstract}
Resumo
Visando verificar o tempo de viabilidade das células espermáticas do cão, na temperatura de $38^{\circ} \mathrm{C}$, e comparar a eficiência de diferentes diluentes na sua criopreservação, foram realizadas 40 colheitas através da manipulação peniana para, no primeiro experimento, realizar o teste de termorresistência (TTR) do sêmen fresco, onde se observou motilidade e vigor espermático, a cada 15 minutos, durante duas horas. No segundo experimento, as amostras obtidas foram diluídas em leite desnatado (LD), Tris+gema de ovo de galinha (TGG) e Tris+gema de ovo de codorna (TGC), refrigeradas a $5^{\circ} \mathrm{C} \mathrm{e,} \mathrm{em} \mathrm{seguida,} \mathrm{adiciona-}$ das de glicerol $(7 \%)$, envasadas e congeladas $\left(-196^{\circ} \mathrm{C}\right)$. O TTR demonstrou que a motilidade e o vigor decaíram gradativamente e, após 45 minutos, observou-se uma redução mais acentuada destes parâmetros espermáticos. Em contrapartida, após o congelamento, verificou-se motilidade de 11,92 \%, 41,54 \% e 50,00 \% e 1,23, 2,77 e 3,00 de vigor espermático, demonstrando diferença significativa $(P \leq 0,05)$ entre o primeiro grupo e os outros dois, que, por sua vez, não diferiram entre si, além de 17,00 $\%, 11,35 \%$ e 10,95 \% de patologias do acrossoma, para os grupos LD, TGG e TGC, respectivamente. Conclui-se, portanto, que o sêmen da espécie canina até 45 minutos a $38^{\circ} \mathrm{C}$ pode ser utilizado na inseminação artificial ou criopreservação e que o diluente Tris, acrescido de gema de ovo (galinha ou codorna), determina melhor viabilidade espermática, pós-descongelamento, quando comparado ao leite desnatado.
\end{abstract}

Palavras-chave: TTR, leite desnatado, Tris-gema.

\begin{abstract}
Aiming to verify the time of viability of the dog's spermatic cells, at a temperature of $38^{\circ} \mathrm{C}$, and to compare the efficiency of different diluents in cryopreservation, a total of 40 collections were accomplished through the manual penile stimulation so, in the first experiment, the term-resistance test (TRT) of the fresh semen could be accomplished, where motility and spermatic vigor were observed, every 15 minutes, for two hours. In the second experiment, the obtained samples were diluted in skimmed milk (SM), Tris+chicken egg yolk (TCEY) and Tris+quail egg yolk (TQEY), refrigerated at $5^{\circ} \mathrm{C}$ and, soon after, added to glycerol (7\%), tubed and frozen $\left(-196^{\circ} \mathrm{C}\right)$. The TRT demonstrated that motility and vigor decayed gradually and, after 45 minutes, a more accentuated reduction of these spermatic parameters was observed. However, after the freezing, a motility of $11.92 \%, 41.54 \%$ and $50.00 \%$ and $1.23,2.77$ and 3.00 of spermatic vigor was verified, demonstrating significant difference $(P \leq 0,05)$ between the first group and the other two, which then, did not differ between each other, besides $17,00 \%, 11,35 \%$ and $10,95 \%$ of acrossome pathologies, for the SM, TCEY and TQEY groups, respectively. Therefore, it is concluded that the semen of the canine species up to 45 minutes at $38^{\circ} \mathrm{C}$ can be used in the artificial insemination or cryopreservation and that the tris diluent added to egg yolk (chicken or quail) determines better spermatic viability, thawing, when compared to the skimmed milk.
\end{abstract}

Keywords: TRT, skimmed milk, Tris-egg yolk.

\section{Introdução}

O sucesso no congelamento do sêmen está diretamente relacionado à qualidade do ejaculado obtido e à técnica de conservação empregada, ambos decisivos nos índices de concepção a serem alcançados através da inseminação artificial (I.A.). Desta forma, o ajuste nos constituintes dos diluidores para o congelamento do sêmen na espécie canina é um fator que oferece a possibilidade de aumentar os índices reprodutivos, solucionando problemas de variação individual do sêmen entre os reprodutores e sua capacidade de sobrevivência após congelamento (Rodrigues e Rodrigues, 1998).

A inseminação artificial, utilizando sêmen congelado, vem-se tornando uma biotécnica usual na espécie canina. No entanto, o tempo decorrido entre a colheita das amostras e a sua utilização na I.A. ou no processo de criopreservação do sêmen pode determinar diferenças na sua viabilidade (Silva et al., 2000).

\footnotetext{
* Departamento de Medicina Veterinária da Universidade Federal Rural de Pernambuco. Av. Dom Manoel de Medeiros s/n, Dois Irmãos, Recife-PE, 52171-900. E-mail: mpguerra@hotlink.com.br
} 
No sêmen bovino, o teste de termorresistência tem como objetivo verificar a correlação entre a motilidade espermática e a taxa de fecundação do sêmen congelado, permitindo a eliminação prévia de amostras de sêmen que não apresentam, pelo menos, $15 \%$ de espermatozóides com motilidade progressiva após o término do período de incubação (Jondet e Rabadeux, 1976; Jondet et al., 1978). Na espécie ovina, no entanto, Evans e Maxwell (1987) recomendam a utilização do sêmen que apresenta, no mínimo, $30 \%$ de motilidade após a utilização do Teste de Termorresistência Lento (cinco horas de incubação a $37^{\circ} \mathrm{C}$ ).

Por outro lado, os diluentes são utilizados para melhorar a viabilidade do sêmen de animais subférteis (Blanchard et al., 1987), aumentar o volume da dose inseminante (Pickett e Amann, 1987), auxiliar na avaliação do sêmen (Kenney et al., 1983) e prolongar a vida dos espermatozóides, protegendoos de condições ambientais desfavoráveis como as variações de temperatura (Pickett e Amann, 1987; Brinsko e Varner, 1992), em virtude dos danos celulares causados pelo frio estarem freqüentemente relacionados às alterações da pressão osmótica durante a criopreservação do sêmen (Lagares et al., 1994; Kohne, 1995).

Desta forma, experimentos têm sido realizados com o objetivo de avaliar a eficiência dos diluentes utilizados na criopreservação do sêmen de cão (Concannon e Battista, 1989; Olar et al., 1989; Silva et al., 1997; Silva et al., 2000). Todavia, a maioria dos pesquisadores utilizam diluidores à base de gema de ovo, tampão Tris e/ou citrato de sódio acrescido de frutose, glucose ou lactose (Andersen, 1975; England, 1993; Günzel-Apel, 1994; Silva et al., 2000), sob diferentes concentrações do glicerol (Andersen, 1975; England, 1993; GünzelApel, 1994; Silva et al., 2000). Muitos diluidores à base de gema de ovo e leite têm sido descritos na literatura para a refrigeração do sêmen de cão (Province et al., 1984; Mies Filho, 1987).

Entretanto, o ovo de codorna tem sido motivo de discussão em virtude de possuir grande valor protéico (Vilar, 1992) e $11 \%$ de gordura (Perez e Perez, 1966), diferindo da composição bioquímica do ovo de galinha, principalmente pela presença do ácido ascórbico - Vitamina C (Fabichak, 1987) e de grandes quantidades de vitaminas $A, D, E, H, B 1$ e o fator PP (Vilar, 1992).

Diante do exposto, este trabalho teve como objetivo avaliar, através do TTR, a viabilidade do sêmen de cão visando sua posterior utilização na inseminação artificial ou criopreservação e comparar a eficiência dos diluentes leite desnatado e Tris acrescido de gema de ovo (galinha ou codorna) na criopreservação do sêmen de cão.

\section{Material e métodos}

Foram utilizados 10 cães de diferentes raças, além de dois SRD, sexualmente maduros, com idade variando entre dois e seis anos. Os cães SRD foram mantidos no canil da UFRPE e alimentados com ração "Croc Dog" e "Croc Baby" (Socil Guymarch, Brasil) e os demais com outras rações comerciais, além de água ad libitum. Inicialmente, foram realizados exames andrológicos, vacinação e vermifugação dos animais. O método de colheita utilizado foi a manipulação peniana, em tubos coletores de vidro graduados, pré-aquecidos à temperatura de $37^{\circ} \mathrm{C}$.

No primeiro experimento, para avaliação do sêmen através do TTR, foram colhidas 10 amostras, referentes à segunda fração do ejaculado, pertencentes a 10 cães de diferentes raças, as quais foram colocadas em banho-maria a $38^{\circ} \mathrm{C}$, durante duas horas, e analisadas quanto à motilidade e o vigor espermático, no momento zero e a intervalos de $15 \mathrm{mi}$ nutos, em microscópio de contraste de fase (Zeiss, Germany). A motilidade foi expressa em porcentagem, e o vigor, subjetivamente, numa escala de 0 a 5 .

No segundo experimento, referente à avaliação de três diluentes para a criopreservação, foi realizada uma colheita de sêmen/animal/semana, no canil, com o objetivo de minimizar o estresse do animal, totalizando 30 colheitas. Em seguida, as amostras foram transportadas durante cinco minutos à temperatura $40^{\circ} \mathrm{C}$, até o laboratório, onde foram colocadas em banho-maria $\left(38^{\circ} \mathrm{C}\right)$, para avaliação dos parâmetros macroscópicos (cor, volume, $\mathrm{pH}$ e odor) e microscópicos do sêmen (concentração, motilidade, vigor e morfologia espermática). A motilidade e o vigor foram avaliados da mesma forma descrita no primeiro experimento, em quatro momentos: M0 (após a colheita), M1 (após refrigeração), M2 (imediatamente após o congelamento) e M3 (uma semana após o congelamento). Para o cálculo da concentração utilizou-se a câmara de Newbauer e, em seguida, calculou-se o número de espermatozóides viáveis/ejaculado. Nos momentos AO e A3 analisou-se a morfologia do acrossoma espermático através da câmara úmida (Mies Filho, 1987), onde foram contadas, no mínimo, 200 células, em microscópio de contraste de fase (Zeiss, Germany), sob aumento de 1000x.

Após a colheita e avaliação, calculou-se o volume do diluente a ser utilizado, de forma que cada dose inseminante possuísse, em média, 300 milhões de espermatozóides. A seguir, o sêmen foi dividido em três frações, de acordo com o grupo experimental. A primeira fração foi diluída com a metade do volume do leite desnatado (LD), a segunda fração com metade do diluente à base de Tris $+20 \%$ de gema de ovo de galinha (TGG) e a terceira fração com metade do Tris $+20 \%$ de gema de ovo de codorna (TGC). Em seguida, as frações foram transferidas para o congelador, onde permaneceram durante uma hora, tempo necessário para atingirem a temperatura de $5^{\circ} \mathrm{C}$. Após o resfriamento, procedeu-se à adição da segunda fração dos diluentes (LD, TGG ou TGC), acrescida de glicerol a $14 \%$, em três partes iguais, a intervalos de 10 minutos. O sêmen, então, foi envasado em 12 minitubos $(0,25$ $\mathrm{mL}$ ) identificados, vedados, colocados em racks, transferidos para o vapor de nitrogênio líquido (distância de $5 \mathrm{~cm}$ ) durante 10 minutos, mergulhados no nitrogênio e transferidos para o botijão criobiológico a $-196^{\circ} \mathrm{C}$. Imediatamente após o congelamento e uma semana depois, dois minitubos de cada amostra (LD,TGG e TGC) foram descongelados em banhomaria $\left(40^{\circ} \mathrm{C}\right)$, durante 60 segundos, para análise de motilidade e vigor espermáticos, assim como morfologia do acrossoma.

Para análise dos resultados, utilizou-se a estatística descritiva e o cálculo de comparação entre as médias para os parâmetros motilidade, vigor e patologia do acrossoma entre os tratamentos (LD, TGG e TGC), através do programa estatístico Copyright ${ }^{\circ}$ (1990), sendo os resultados considerados significativos a $5 \%$. 


\section{Resultados e discussão}

No primeiro experimento, a resistência do espermatozóide de cão à temperatura de $38^{\circ} \mathrm{C}$, avaliada através do TTR (Tabela 1), a intervalo de 15 minutos, demonstrou que as células espermáticas apresentam, aos 45 minutos, $82,50 \%$ de motilidade e 4,64 de vigor. Apesar da literatura não se referir à utilização deste teste, de acordo com Willard et al. (1994) o sêmen de cão pode ser manipulado, à temperatura ambiente, por aproximadamente 15 minutos, sem afetar a sua qualidade, que deve apresentar mais de $80 \%$ de motilidade.

Tabela 1: Percentual de motilidade e vigor espermático no sêmen de cão submetido ao TTR, à temperatura de $38^{\circ} \mathrm{C}$, e avaliados a intervalos de 15 minutos

\begin{tabular}{ccc}
\hline Minutos & Motilidade (\%) & Vigor (0-5) \\
\hline $\mathbf{0}$ & 100,00 & 5,00 \\
$\mathbf{1 5}$ & 95,00 & 4,86 \\
$\mathbf{3 0}$ & 87,86 & 4,71 \\
$\mathbf{4 5}$ & 82,50 & 4,64 \\
60 & 77,14 & 4,57 \\
$\mathbf{7 5}$ & 68,57 & 4,14 \\
90 & 57,14 & 3,43 \\
105 & 48,57 & 2,86 \\
120 & 32,86 & 2,29 \\
\hline
\end{tabular}

Desta forma, os resultados demonstram que o sêmen da espécie canina pode permanecer até 45 minutos à temperatura de $38^{\circ} \mathrm{C}$, período suficiente para a realização dos preparativos da fêmea e do material utilizado para inseminação artificial ou criopreservação, tendo em vista que os valores obtidos são considerados parâmetros satisfatórios para inseminação com o sêmen de reprodutores da espécie canina, de acordo com Willard et al. (1994) e Inamassu et al. (1999). Entretanto, não se pode comparar os resultados obtidos com os evidenciados por Silva et al. (2000), em virtude destes autores não avaliarem a motilidade e o vigor espermático após 30 minutos, período decorrido entre a colheita e o processamento do sêmen, correspondendo ao transporte do sêmen até o laboratório, nem com as normas do Manual para Exame Andrológico e Avaliação do Sêmen Animal (1998), tendo em vista que na espécie bovina, o TTL utiliza cinco horas, diferindo da duração utilizada neste experimento, que foi de duas horas.

Outro aspecto que deve ser relacionado é que os dados obtidos in vitro, neste experimento, não foram avaliados in vivo através da taxa de fecundação, segundo recomendação de Jondet e Rabadeux (1976) e Jondet et al. (1978), ao utilizarem o TTR na espécie bovina, nem com os de Evans e Maxwell (1987), ao recomendarem a utilização do sêmen da espécie ovina que apresentassem, no mínimo, 30\% de motilidade após o TTL.

No segundo experimento, referente ao processo de refrigeração e congelação, observou-se coloração branco-opalescente, sem odor, volume médio de $12 \mathrm{~mL}$ referente à segunda fração do ejaculado e $\mathrm{pH} 6,7$. No entanto, os valores microscópicos obtidos através da análise das amostras do sêmen de cães encontram-se na Tabela 2, onde se observa, imediatamente após a colheita, motilidade de 95\% e 4,77 de vigor, valores considerados de excelente qualidade, de acordo com Dobrinsky et al. (1993), Willard et al. (1994), Silva e Verstegen (1995), Moura et al. (1999) e Silva et al. (2000).

No entanto, constata-se que no sêmen refrigerado ao se utilizar o leite desnatado como diluente (LD), evidenciou-se diferença significativa $(P £ 0,05)$ no baixo percentual de motilidade espermática $(69,23 \%)$, quando comparada àquelas observadas ao adicionar Tris acrescido de gema de ovo de galinha $(85,38 \%)$ ou ovo de codorna $(88,08 \%)$. Da mesma forma, o vigor obtido no grupo LD $(3,00)$ também foi inferior $(P \leq 0,05)$ àqueles constatados nos grupos TGG $(3,85)$ e TGC $(4,38)$, demonstrando que a $5^{\circ} \mathrm{C}$, a ação crioprotetora do diluente à base de leite desnatado sobre a célula espermática é inferior àquela exercida pela gema de ovo de galinha ou codorna, discordando dos achados de Province et al. (1984) e Mies Filho (1987), que relataram que o diluidor à base de leite desnatado, por ser de fácil preparo, menor custo e capaz de preservar a célula espermática, pode substituir o diluidor a base de gema de ovo. Por outro lado, corrobora com Andersen (1975), England (1993), Günzel-Apel (1994) e Moura et al. (1999).

Tabela 2: Percentual de motilidade e vigor espermático no sêmen da espécie canina, com o sêmen fresco ( $\mathrm{m} 0$, $\mathrm{v} 0)$, refrigerado $(\mathrm{m} 1, \mathrm{v} 1)$, imediatamente após o congelamento ( $\mathrm{m} 2$, v2) e uma semana após o congelamento $(\mathrm{m} 3, \mathrm{v} 3)$.

\begin{tabular}{lllllllll}
\hline \multicolumn{1}{c}{ Grupo Experimental } & m0 & v0 & m1 & v1 & m2 & v2 & M3 & v3 \\
\hline Leite desnatado (LD) & 95,00 & 4,77 & $69,23^{\mathrm{a}}$ & $3,00^{\mathrm{a}}$ & $24,23^{\mathrm{a}}$ & $1,85^{\mathrm{a}}$ & $11,92^{\mathrm{a}}$ & $1,23^{\mathrm{b}}$ \\
Tris+gema de ovo de galinha (TGG) & 95,00 & 4,77 & $85,38^{\mathrm{b}}$ & $3,85^{\mathrm{b}}$ & $58,46^{\mathrm{b}}$ & $3,31^{\mathrm{b}}$ & $41,54^{\mathrm{b}}$ & $2,77^{\mathrm{b}}$ \\
Tris+gema de ovo de codorna (TGC) & 95,00 & 4,77 & $88,08^{\mathrm{b}}$ & $4,38^{\mathrm{b}}$ & $63,43^{\mathrm{b}}$ & $3,46^{\mathrm{b}}$ & $50,00^{\mathrm{b}}$ & $3,00^{\mathrm{b}}$ \\
\hline
\end{tabular}

Letras diferentes indicam diferença estatística $(P \leq 0,05)$.

Um fato que também pode ser observado na Tabela 2 é que, apesar de não haver diferenças estatísticas, os resultados de motilidade e vigor espermático, ao se utilizar o ovo de codorna, foram superiores aos obtidos com o diluente à base de ovo de galinha em todos os momentos, o que pode ter sido determinado pela presença de vitaminas, como o ácido ascórbico (Fabichak, 1987) ou pelo baixo teor de gordura (Perez e Perez, 1966) encontrados na gema de ovo de codorna. 
Por conseguinte, acredita-se que a gema de ovo (galinha ou codorna) potencializa o efeito crioprotetor do glicerol sobre a membrana plasmática dos espermatozóides, quando submetidos a temperaturas abaixo de $0^{\circ} \mathrm{C}$, ratificando os achados de England (1993) ao descreverem as propriedades crioprotetoras do glicerol e da gema de ovo de galinha em proteger a integridade das membranas espermáticas durante o procedimento de criopreservação, bem como os relatos de Vilar (1992) ao destacar as propriedades do ovo de codorna que, provavelmente, minimizam os efeitos das alterações da pressão osmótica, decorrentes da ação do frio sobre as estruturas espermáticas, evidenciadas por Lagares et al. (1994) e Kohne (1995).

Em contrapartida, ao se avaliar as patologias do acrossoma (Tabela 3), constata-se que houve aumento no percentual destas patologias espermáticas após o procedimento de criopreservação (A3). No entanto, não foram evidenciadas diferenças estatísticas entre os grupos experimentais, acreditando-se, portanto, que os diluentes LD, TGG ou TGC, provavelmente por apresentarem propriedades bioquímicas compatíveis com a célula, não danificam o acrossoma, mantendo, portanto, a capacidade fertilizante da célula espermática do cão após o congelamento, o que possibilitará a sua utilização na I.A., tendo em vista que Concannon e Battista (1989), Olar et al.

\section{Referências}

ANDERSEN, K. Insemination with frozen dog semen based on a new insemination technique. Zuchthygiene, v. 10, p. 1-4, 1975.

BLANCHARD, T. L.; VARNER, D. D.; LOVE, C. C.; HURTGEN, J. P.; CUMMINGS, M. R.; KENNEY, R. M. Use of an extender containing antibiotic to improve the fertility of a stallion with seminal vesiculitis due to Pseudomonas aeruginosa. Theriogenology, v. 28, n. 4, p. 541-546, 1987.

BRINSKO, S. P.; VARNER, D. D. Artificial insemination and preservation of semen. In: BLANCHARD, T. L., VARNER, D. D. (Ed.) Staillon management. Vet. Clin. North America: Equine Practice, v. 8, n. 1, p. 205-218, 1992.

CBRA - Colégio Brasileiro de Reprodução Animal. Manual para exame andrológico e avaliação do sêmen animal, 2. ed., Belo Horizonte, 1998.

CONCANNON, P. W.; BATTISTA, M. Canine semen freezing and artificial insemination. In: KIRK, R. W. (Ed.) Current veterinary therapy - Small animal practice, 10. ed. Philadelphia: W. B. Saunders, 1989, p. 12471259.

DOBRINSKY, I.; LULA, I.; BARTH, A.D. et al. Effects of four different extenders and three different freezing rates on post-thaw viability of dog semen. J. Reprod. Fertil., v. 47, p. 291-286, 1993.

ENGLAND, G. C. W. Cryopreservation of dog semen: Review. J. Reprod. Fertil., v. 47 (suppl), p. 243-255, 1993.

EVANS, G., MAXWELL, W.M.C. (Ed.) Salamon's artificial insemination of sheep and goats. Sydney: Butterworths, 1987.

FABICHAK, I. (Ed.) Codorna: criação, instalação e manejo. São Paulo: Nobel, 1987.

GÜNZEL-APEL, A. R. Fertilitätskontrolle und Samenübertragung beim hund. Hannover: Gustav Fischer Verlag Jena, 1994, cap. 1, p. 20-31: Der Zuchtrüde. Cap. 3, p.8 4: Insytrumentelle Samennübertragung.

INAMASSU, A.; UECHI, E.; LOPES, M.D. Viabilização do teste hipoosmótico em cães e sua relação com outras variáveis espermáticas. Rev. Bras. Reprod. Anim., v. 23, n. 3, p. 302-304, 1999.
(1989) e Silva et al. (1997) relacionam a eficácia do diluente ao sucesso da inseminação artificial.

Tabela 3: Percentual de patologias do acrossoma do sêmen de cão submetido ao procedimento de criopreservação, no sêmen fresco (A0) e uma semana após o congelamento (A3).

\begin{tabular}{lcc}
\hline Grupo Experimental & A0 & A3 \\
\hline Leite desnatado (LD) & 6,97 & 17,00 \\
Tris+gema de ovo de galinha (TGG) & 6,97 & 11,35 \\
Tris+gema de ovo de codorna (TGC) & 6,97 & 10,95 \\
\hline
\end{tabular}

\section{Conclusões}

Conclui-se, portanto, que o sêmen da espécie canina submetido à temperatura de $38^{\circ} \mathrm{C}$ durante 45 minutos, apresenta excelente qualidade para ser utilizado na inseminação artificial ou criopreservação e que o diluente Tris acrescido de gema de ovo (galinha ou codorna) determina melhor viabilidade espermática, pós-descongelamento, quando comparado ao leite desnatado.

JONDET, R.; RABADEUX, Y. Thermorresistence dans apreciation de la valeur du sperm congélé de taureau. Elevage et Insemination, n. 156, p. 13-19, 1976.

JONDET, R.; MIES FILHO, A., RABADEUX, Y. Lépreuve de thermorésistance dans l'appréciation de la valeur du sperm bovin congelé. Comptes rendus des sénas de la Société de Biologie, v. 4, p. 764, 1978.

KENNEY, R.M.;HURTGEN, J.;PIERSON, R.; WITHERSPOON, D.; SIMONS, J. Manual for Clinical fertility evaluation of the stallion. Hastings, EUA. Soc. For Theriogenology, 1983, p. 354.

KOHNE, K. Einsatz osmotischer Resistezbestimmungen zur beuurteilung der flüssig - und kryokonservierungsfähigkeit von hengstspermatozoen. Tese (Doutorado em Medicina Veterinária), Hannover, Tierärztl. Hochsch, 1995.

LAGARES, M. A.; PETZOLDT, R.; SIEME, H.; KLUG, E. Zur hypoosmotischen belastborkeit von spermien des hengstes. Reprod. Dom. Anim., v. 29, p. 204, 1994.

MIES FILHO, A. Reprodução dos Animais. 6. ed., Porto Alegre: Sulina, 1987.

MOURA, C.S.; CAVALCANTI, M.C.O.; GUERRA, M. M .P.; TAVARES, P. T. S. Criopreservação do sêmen canino utilizando diferentes métodos de refrigeração. Rev. Bras. Reprod. Anim., v. 23, n. 3, p. 304-306, 1999.

OLAR, T.T.; BOWEN, R. A.; PICKETT, B. W. Influence of extender, cryopreservative and seminal processing procedures of postthaw motility of canine spermatozoa frozen in straws. Theriogenology, v. 31, n. 2, p. 451-461, 1989.

PEREZ, F., PEREZ, F. Coturnicultura: tratado de cría y exploración industrial de codornices. Barcelona: Científico-Médica, 1966.

PICKETT, B. W.; AMANN, R. P. Extension and storage of stallion spermatozoa: a review. J. Eq. Vet. Sci., v. 7, n. 5, p. 289-302, 1987.

PROVINCE, A. C.; AMANN, R. P.; PICKETT, B. W.; SQUIRES, E. L. Extenders for preservation of canine and equine spermatozoa at $5^{\circ} \mathrm{C}$. Theriogenology, v. 4, p. 409-415, 1984. 
RODRIGUES, B. A.; RODRIGUES, J. L. Efeito da adição de diferentes concentrações de albumina sérica bovina (BSA) ao diluidor a base de TRIS sobre a viabilidade in vitro do sêmen canino criopreservado. Arq. Fac. Vet. UFRGS, n. 26/2, p. 32-49, 1998.

SILVA, A.R.; CARDOSO, R.C.S.; SILVA, L.D.M. Congelação de sêmen canino com diferentes concentrações de gema de ovo e glicerol em diluidores à base de Tris e água de coco. Cienc. Rural, v. 30, n. 6, p. 1021-1025, 2000.

SILVA, A. R.; CARDOSO, R. C. S.; SOUZA, D. M. B.; SILVA, L. D. M. Comparação entre os diluentes à base de água de coco e à base de Tris na criopresenvação de sêmen canino. SIMPÓSIO NACIONAL DE
BIOTECNOLOGIADAREPRODUÇÃO DE ANIMAIS DOMÉSTICOS, 2., 1997, Fortaleza. Anais..., 1997. p.

SILVA, L.D.M.; VERSTEGEN, J.P. Comparisons between three different extenders for canine intrauterine insemination with frozen-thawed spermatozoa. Theriogenology, v. 44, p. 571-579, 1995.

VILAR, E. A. Codorna: Manejo correto - lucro certo. Manual prático. 1992, p. 9-12.

WILLARD, M. D.; TVEDTEN, H.; TURNWALD, G.H. Small animal clinical diagnosis by laboratory methods. 2. ed. Philadelphia: W.B. Daunders Company, 1994. 